“ (C) 2016 IEEE. Personal use of this material is permitted. Permission from IEEE must be obtained for all other uses, in any current or future media, including

reprinting/republishing this material for advertising or promotional purposes, creating new collective works, for resale or redistribution to servers or lists, or reuse of any copyrighted component of this work in other works." 


\title{
A Comparative Study of Battery Balancing Strategies for Different Battery Operation Processes
}

\author{
Linfeng Zheng ${ }^{1,2}$, Jianguo Zhu ${ }^{1}$, Guoxiu Wang ${ }^{2}$ \\ 1. Faculty of Engineering and Information Technology, University of Technology Sydney, Sydney, N.S.W., 2007, Australia \\ 2. Centre for Clean Energy Technology, University of Technology Sydney, Sydney, N.S.W., 2007, Australia
}

Linfeng.Zheng@student.uts.edu.au

\begin{abstract}
To reduce the effect of cell inconsistencies and improve battery pack capacity, battery balancing techniques are essentially required in battery management systems (BMSs). This paper presents a comparative study of four battery balancing strategies for different battery operation processes. These balancing strategies are developed from the state-of-theart battery balancing circuits and algorithms reported in recent literature. The performance of balancing strategies is evaluated and compared by battery pack maximum available capacity, state of charge (SOC) variances at the end of charge (EOC) and end of discharge (EOD). The comparative result is helpful for BMSs developers to employ optimal balancing strategies in actual applications.
\end{abstract}

Keywords-Battery balancing, battery pack capacity, SOC variance, battery management system (BMS).

\section{INTRODUCTION}

As one single battery cell has limited capacity and voltage, it normally needs up to hundreds, even thousands of single cells connected in series and parallel for electrified vehicles and energy storage systems to provide sufficient power and energy. In such a battery pack, inevitable cell inconsistencies are concerned due to two categories: the inconsistent manufacturing process such as different internal impedance and self-discharge rate, and the inhomogeneous operating environment such as various ambient temperature $[1,2]$. To improve cell consistencies, increase battery pack capacity and prolong battery pack service life, battery cell balancing techniques are essentially required in battery management systems (BMSs).

Among the literature, most papers focus on battery balancing circuit topologies [2-7] and balancing algorithms [1, 5-14]. The battery balancing circuit topologies reported in [27] mainly include resistor bleeding (passive balance), cell to cell, cell to pack, pack to cell and cell to pack to cell, and their advantage and disadvantage were presented in detail. According to the literature [1, 5-14], the battery balancing algorithms can be mainly divided into two categories: voltage-based balancing algorithm and SOC-based balancing algorithm. The voltage-based balancing algorithm which targets the consistent battery cell terminal voltages is widely used in research and industry [5-10]. The SOC-based balancing algorithm which targets the consistent battery cell SOCs requires accurate cell SOC estimation [1, 10-14]. While their pros and cons are discussed in the literature, unfortunately, there are few studies that compare these balancing circuits incorporating the balancing algorithms in a comprehensive and systematical manner.

Regardless of balancing circuits and algorithms, when to perform balancing plays a vital role in balancing performance. Performing balancing during different battery operation processes, such as constant current charging processes, dynamic current discharging processes and rest time, may lead to various balancing effects.

Therefore, it is essential to carry out a comparative study of battery balancing strategies incorporating balancing circuits and algorithms for different battery operation processes.

The rest of the paper is organized as follows: Section II introduces four battery balancing strategies which are developed from the balancing circuits and balancing algorithms for simulation experiment. The experimental results and evaluation of the four balancing strategies for different battery operation processes are discussed and reported in Section III, followed by the conclusions and future work in Section IV.

\section{BATTERY BALANCING STRATEGIES}

According to the balancing current used for battery cell charging or discharging, we classify battery balancing circuits into four categories: passive discharge balance, active discharge balance, charge balance, and charge-discharge (namely bidirectional) balance. Regardless of balancing circuits, effective balancing algorithms are highly required to maximize balancing effects. It has been reported that the voltage-based balancing algorithm cannot achieve desirable

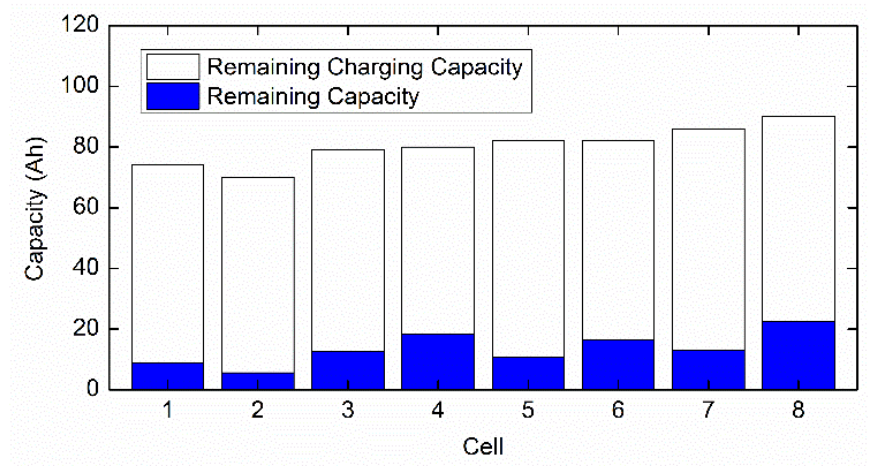

Fig. 1. Initial cell remaining capacities and remaining charging capacity. 


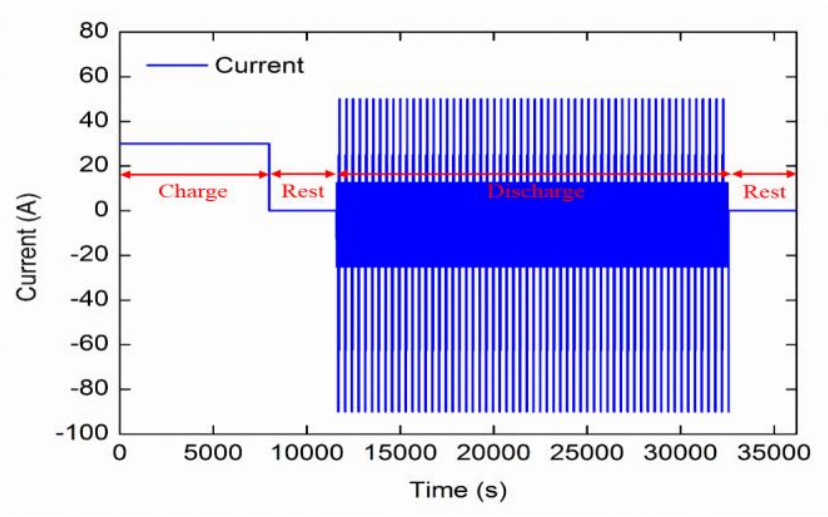

Fig. 2 Battery operation processes.

balancing results because a small voltage variation may lead to a large capacity inconsistency, especially for some kinds of batteries with flat charge/discharge voltage plateau [1]. The SOC-based algorithm can really reflect the battery capacity level and therefore it was employed for this study. Combining the classified circuit categories with the SOC-based balancing algorithm, we can obtain four balancing strategies: SOCbased passive discharge balance (PDB), SOC-based active discharge balance (ADB), SOC-based charge balance (CB), and SOC-based charge-discharge balance (CDB).

\section{RESULTS AND DISCUSSION}

In order to investigate the performance of different balancing strategies applied for various battery operation processes, eight Lithium manganite battery cell models connected in series with different available capacities and SOCs shown in Fig. 1 were used for balancing experimental simulation. The charge/discharge balance current was set to 4 A while the balance referenced SOC was set to the average SOC with a band of $+/-1 \%$. The efficiency of the balancer was set to $85 \%$, which means that there are $15 \%$ of balance energy will be dissipative during balancing.

The battery operation processes usually include charge process, rest time after charge, discharge process, and rest time after discharge. To simulate the charge and discharge processes of battery used in electric vehicles, a constant current was used for battery charging and dynamic stress test (DST) [15] profiles were used for battery discharging, as shown in Fig. 2. Each battery operation cycle began with a constant current at $\mathrm{C} / 3$ to charge to the battery pack until one of the battery cells reached to the upper limit voltage $4.2 \mathrm{~V}$. Then there was a rest for $1 \mathrm{~h}$ followed by DST profiles to discharge the battery pack until one of the battery cells reached the lower limit voltage $3 \mathrm{~V}$. Subsequently, it followed a rest for $1 \mathrm{~h}$ and then repeated the operation cycle. The battery pack maximum available capacity was calculated by ampere-hour counting during the whole discharge process. Five battery operation cycles were used for evaluating the balancing results including battery pack maximum available capacity, SOC variances at the end of discharge (EOD) and the end of charge (EOC).
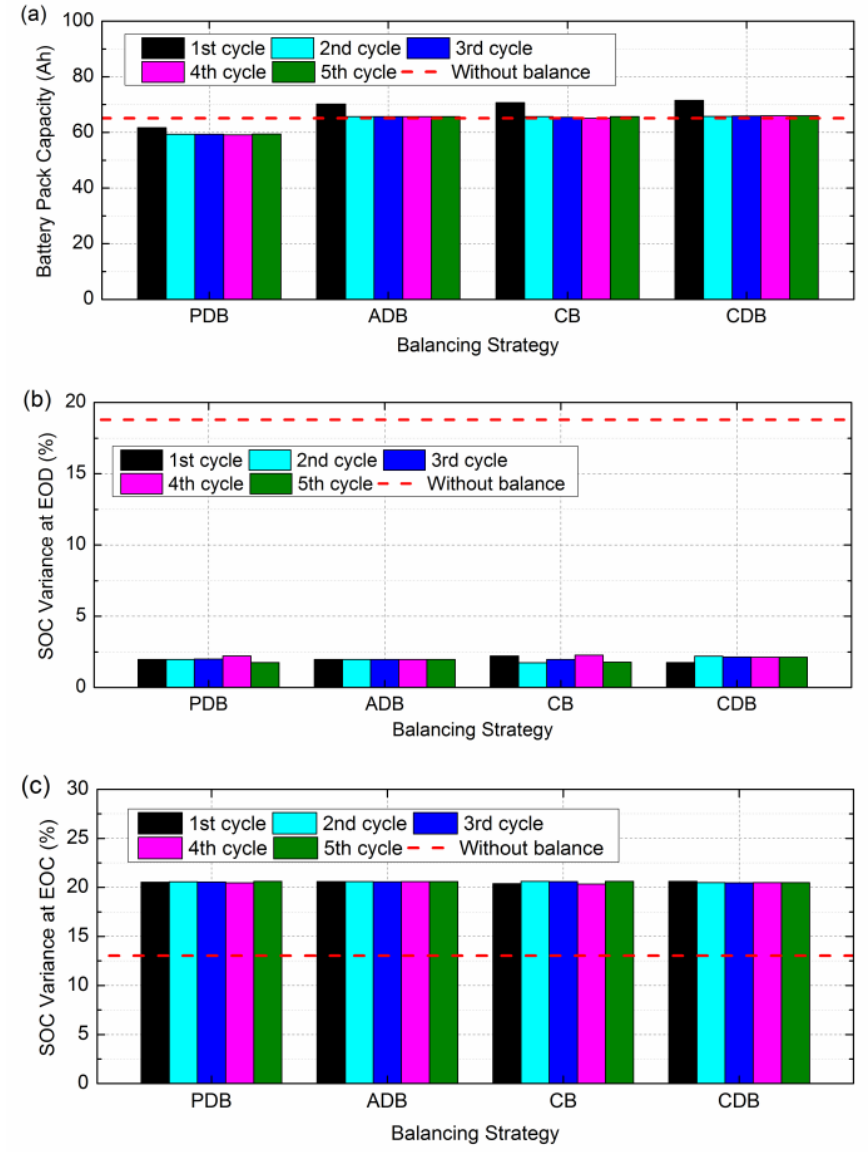

Fig. 3. Battery balancing results when balancing performed during battery discharge processes: (a) battery pack capacity, (b) SOC variance at EOD and (c) SOC variance at EOC.

Without balance, the battery pack maximum available capacity is $65.06 \mathrm{Ah}$ which is less than the minimum cell capacity (cell 2 charged in constant current regime) of 66.63 $\mathrm{Ah}$, while the maximum SOC variance at $\mathrm{EOC}$ is $13.05 \%$ and the maximum SOC variance at EOD is $18.8 \%$. The mean battery cell capacity (charged in constant current regime) is $77.16 \mathrm{Ah}$.

When balancing performed during battery discharge processes, the balancing results are shown in Fig. 3. In Fig. 3(a), the ADB, CB and CDB strategies are able to increase the battery pack capacity, and the final battery pack capacities are a little larger than the capacity without balance. However, the balanced pack capacities were still less than the minimum cell capacity. It is noted that the PDB method caused a counterproductive balancing result that the battery pack capacity is less than the value without balance. This is because that the PDB method is an energy dissipation method and the dissipative energy could not supply from elsewhere.

It can be seen that different balancing strategies were able to make an improvement in decreasing SOC variances at EOD compared with the result without balance as shown in Fig. 3(b). On the contrary, the SOC variances at EOC were increased, which are larger than the value without balance as shown in Fig. 3(c). 

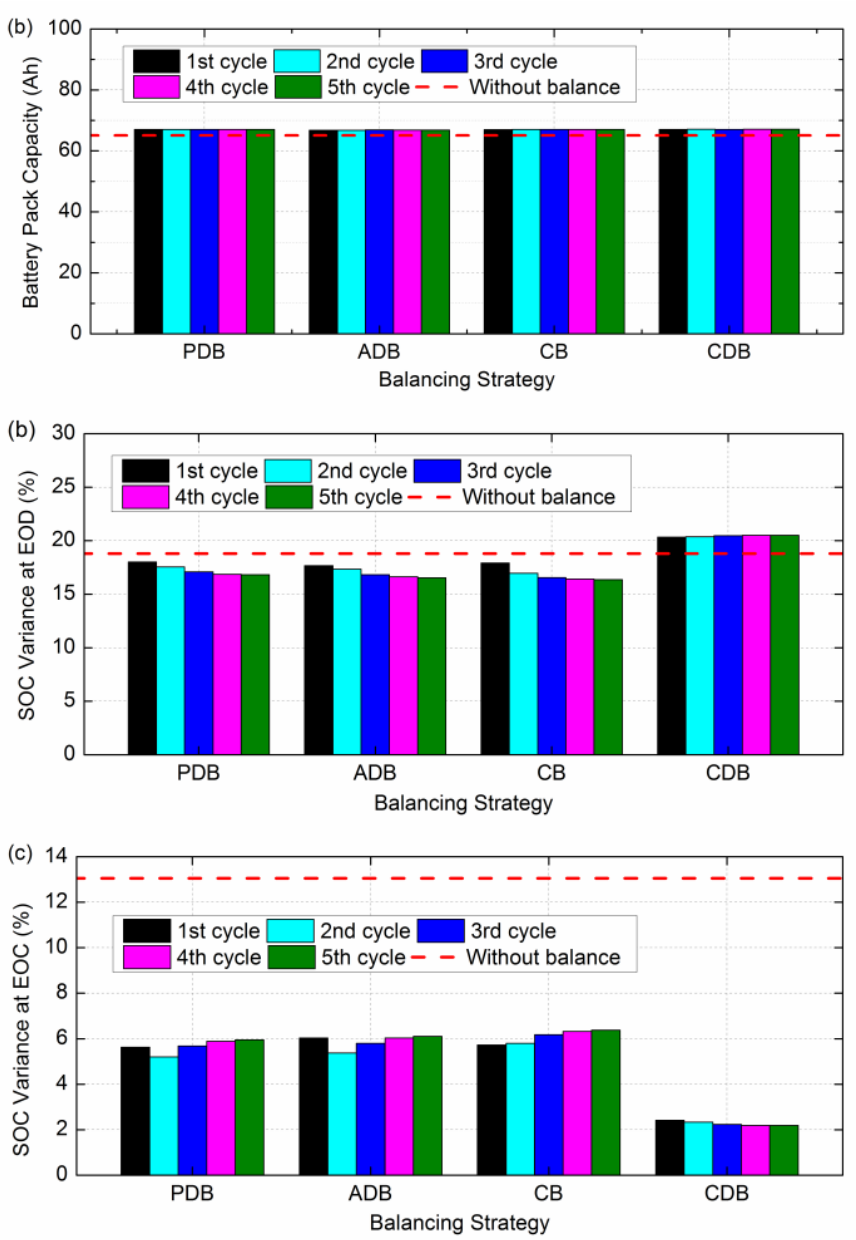

Fig. 4. Battery balancing results when balancing performed during battery charge processes: (a) battery pack capacity, (b) SOC variance at EOD and (c) SOC variance at EOC.

Fig. 4 shows the balancing results when balancing performed during battery charge processes. In Fig. 4(a), four balancing strategies were able to make an improvement on the battery pack capacities, which are about equal to 67.00 Ah. As shown in Fig. 4(c), the SOC variances at EOC were shortened to a small level compared with the value without balance. However, SOC variances at EOD presented inconsistent change. For the $\mathrm{PDB}, \mathrm{ADB}$ and $\mathrm{CB}$ strategies, there was a little decrease on the SOC variances at EOD. But for the CDB strategy, the SOC variance at EOD was increased to be larger than the value without balance.

When balancing performed during battery rest time after discharge, the battery balancing results are shown in Fig. 5. The battery pack capacities for four different balancing strategies depicted in Fig. 5(a) showed a shade of increment in comparison with the value without balance. The SOC variances at EOD appeared a gradual decline trend as shown in Fig. 5(b) and in the final cycle, the SOC variances can be limited to a small value. However, the SOC variances at EOC shown in Fig. 5(c) gradually rose with the cycle time and finally achieve a high level. Moreover, the balancing results of the fifth cycle shown in Fig. 5 are similar to the results
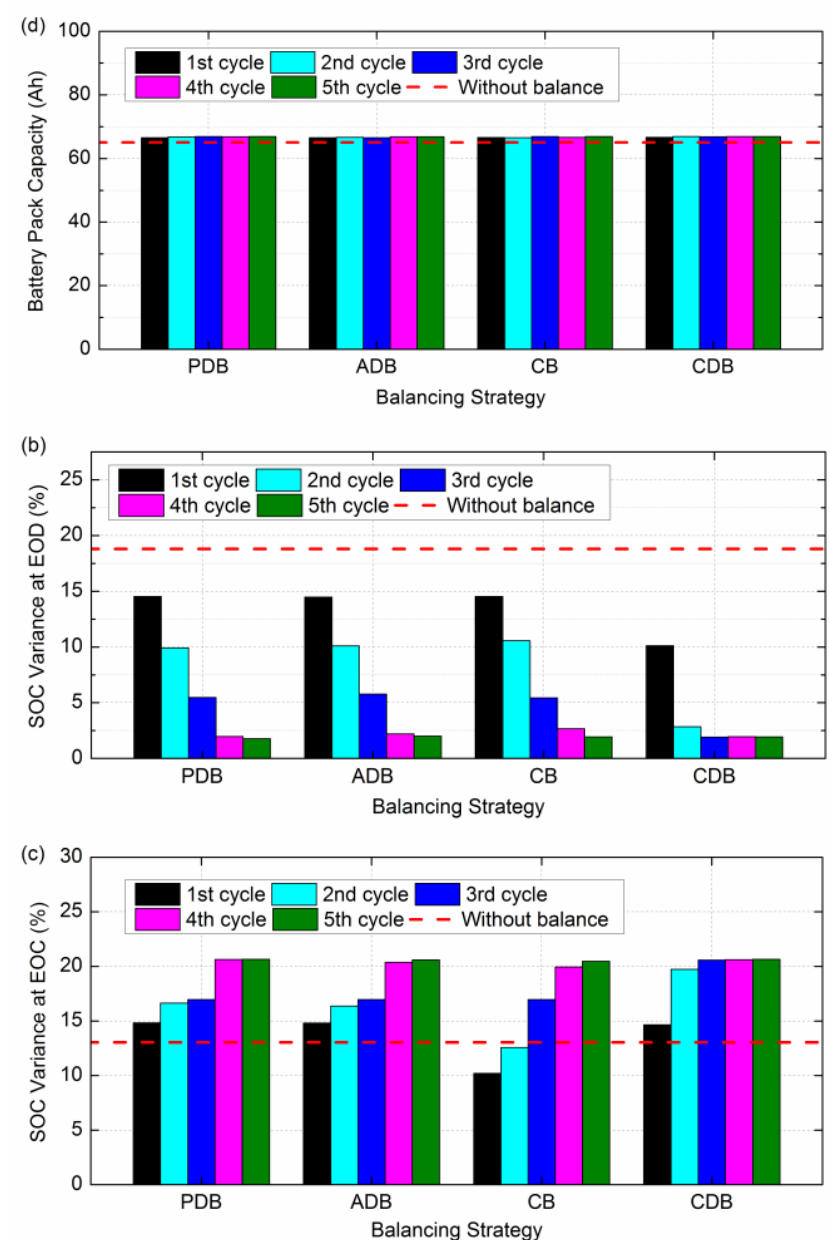

Fig. 5. Battery balancing results when balancing performed during battery rest time after discharge: (a) battery pack capacity, (b) SOC variance at EOD and (c) SOC variance at EOC.

when balancing performed during battery discharge processes shown in Fig. 3. This is because the four mentioned-above balancing strategies are able to limit the SOC variance at EOD in a balance referenced SOC band when balancing performed during battery discharge process and rest time after discharge. However, it would have a counterproductive effect on the variance at EOC and increase the value of the variance at EOC.

The balancing results when balancing performed during battery rest time after charge are drawn in Fig. 6, where battery pack maximum available capacities of the final cycle can be approximately increase to $66.86 \mathrm{Ah}$ which is a little larger than the battery pack capacity value without balance. SOC variances at EOD and EOC showed in Fig. 6(b) and 6(c), respectively, presented exactly opposite results to the balancing results when balancing performed during battery rest time after charge shown in Fig. 5(b) and Fig. 5(c). It can be seen that in Fig. 6(b), the SOC variances at EOD dropped gradually and reached to a small level. But the SOC variances at EOD finally increased to be larger than the value without balance. 

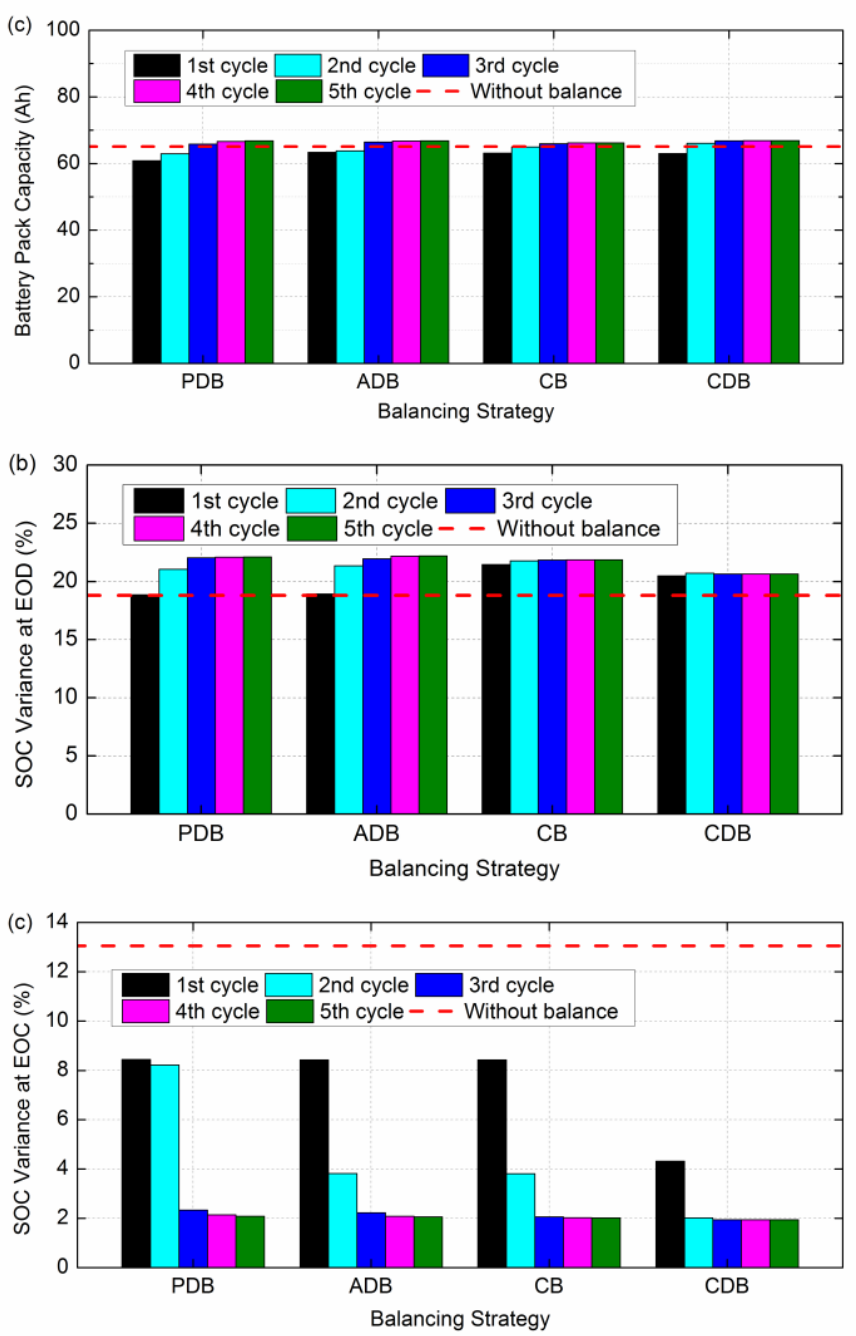

Fig. 6. Battery balancing results when balancing performed during battery rest time after charge: (a) battery pack capacity, (b) SOC variance at EOD and (c) SOC variance at EOC

From above results, it can be summarized that when balancing performed during one kind of battery charge process, rest time after charge, and rest time after discharge, four mentioned-above balancing strategies are able to increase the battery pack capacity to be approximately equal to the minimum cell capacity. During the discharge processes, the PDB method would make a counterproductive balance result which decreases the battery pack capacity, while other balancing strategies can make an improvement in the battery pack capacity but cannot reach the minimum cell capacity.

Besides comparing how much battery pack capacity can be increased, another important factors are SOC variances at EOC and EOD. It would decrease the SOC variance at EOD when balancing performed during battery discharge processes and rest time after discharge, and therefore the battery pack is able to be discharged more capacity. On the contrary, the SOC variance at EOC would be declined to a small value when balancing performed during battery charge processes
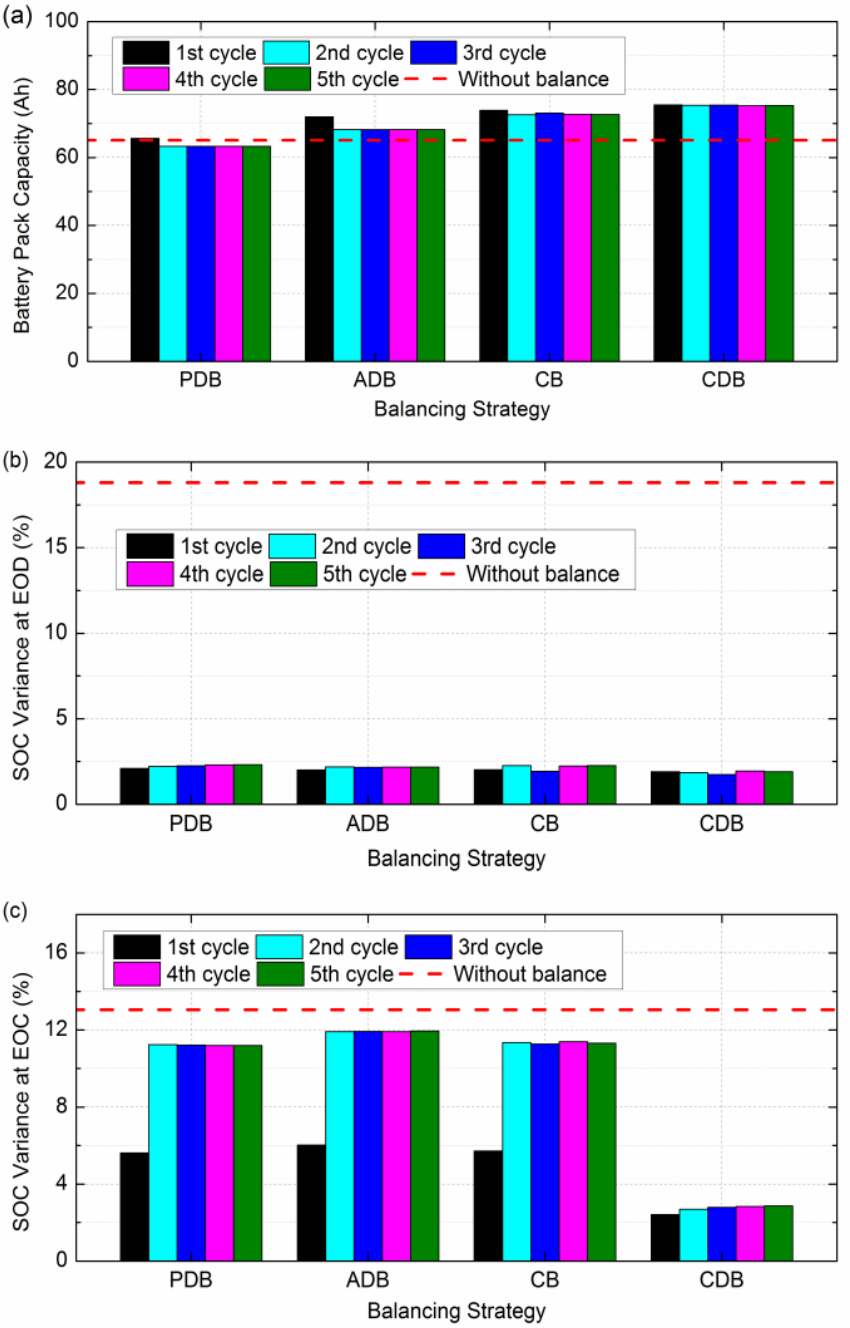

Fig. 7. Battery balancing results when balancing performed during both battery charge and discharge processes: (a) battery pack capacity, (b) SOC variance at EOD and (c) SOC variance at EOC.

and rest time after charge, and therefore the battery pack can be charged more capacity.

The above balanced battery pack capacity can be maximally increased to close to the minimum cell capacity, but it is still much less than the mean battery cell capacity. To maximally utilize the battery pack capacity, namely the mean battery cell capacity, it should fully charge and discharge each cell of the battery pack. In other words, the SOC variances at both EOC and EOD should be maximally decreased to a small level, and therefore it would maximally increase the battery pack maximum available capacity. Thus, the battery balancing should be performed during both battery charge and discharge processes.

Fig. 7 shows the battery balancing results when balancing performed during both battery charge and discharge processes. In Fig. 7(a), the ADB, CB and CDB strategies are able to increase the battery pack capacity to a high value which is more than the minimum cell capacity. Especially for the CDB strategy, the battery pack capacity can be increased to 75.30 
Ah, which improves $15.74 \%$ compared with the capacity without balance $(65.05 \mathrm{Ah})$ and is very closed to the mean battery cell capacity. It is noted that for the CDB strategy, SOC variances at EOC and EOD were able to be significantly dropped to a small level, and therefore the battery capacity of the CDB strategy is larger than other strategies. As for the PDB strategy, although SOC variances at both EOD and EOC can be declined, there was a large amount of energy consumption due to resistor bleeding during battery discharge processes, which caused the battery pack capacity decrement.

\section{CONCLUSION}

Battery balancing techniques are essentially required in BMSs for increasing battery pack capacity and prolonging battery pack service life. Regardless of balancing circuits and algorithms, when to perform balancing plays a vital role in balancing performance. Performing balancing during different battery operation processes would lead to various balancing effects.

In this paper, four battery balancing strategies including $\mathrm{PDB}, \mathrm{ADB}, \mathrm{CB}, \mathrm{CDB}$, were developed from the state-of-theart balancing circuits and balancing algorithms for simulation experiment. During one single kind of battery charge processes, rest time after charge and rest time after discharge, four mentioned-above balancing strategies can increase the battery pack capacity to be closed to the minimum cell capacity. During the battery discharge processes, the PDB strategy would make a counterproductive balance result which decreases the battery pack capacity, while other balancing strategies can make an improvement in the battery pack capacity but cannot reach the minimum cell capacity. To maximally utilize the battery pack capacity, the SOC variances at both EOC and EOD should be maximally decreased to a small value, and therefore it would maximally increase the battery pack capacity. Performing balancing during both battery charge and discharge processes with the $\mathrm{ADB}, \mathrm{CB}$ and $\mathrm{CDB}$ strategies can effectively increase the battery pack capacity to a higher level, which can be approximately close to the mean cell capacity.

Actual battery balancing experiment and energy consumption analysis during battery balancing will be investigated in the future work.

\section{ACKNOWLEDGMENT}

This work was financially supported by the Automotive Australia 2020 Cooperative Research Centre (AutoCRC).

\section{REFERENCES}

[1] Y. Zheng, M. Ouyang, L. Lu, J. Li, X. Han and L. Xu, "On-line equalization for lithium-ion battery packs based on charging cell voltages: Part 1. Equalization based on remaining charging capacity estimation," J. Power Sources, vol. 247, pp. 676-686, 2014.

[2] J. Gallardo-Lozano, E. Romero-Cadaval, M. I. Milanes-Montero and M. A. Guerrero-Martinez, "Battery equalization active methods," J. Power Sources, vol. 246, pp. 934-949, 2014.
[3] F. Baronti, R. Roncella and R. Saletti, "Performance comparison of active balancing techniques for lithium-ion batteries," J. Power Sources, vol. 267, pp. 603-609, 2014.

[4] M. Caspar, T. Eiler and S. Hohmann, "Comparison of active battery balancing systems," in Vehicle Power and Propulsion Conference (VPPC), 2014 IEEE, 2014, pp. 1-8.

[5] S. Li; C. C. Mi and M. Zhang, "A high-efficiency active batterybalancing circuit using multiwinding transformer." Industry Applications, IEEE Transactions on, vol. 49 pp. 198-207, 2013.

[6] M. Daowd; N. Omar, P. Van Den Bossche and J. Van Mierlo, "Passive and active battery balancing comparison based on MATLAB simulation." in Vehicle Power and Propulsion Conference (VPPC), 2011 IEEE, 2011, pp. 1-7.

[7] F. Mestrallet, L. Kerachev, J. Crebier and A. Collet, "Multiphase interleaved converter for lithium battery active balancing," Power Electronics, IEEE Transactions On, vol. 29, pp. 2874-2881, 2014.

[8] C. H. Kim, M. Y. Kim; H. S. Park and G. W. Moon, "A modularized two-stage charge equalizer with cell selection switches for seriesconnected lithium-ion battery string in an HEV." Power Electronics, IEEE Transactions on, vol. 27, pp. 3764-3774, 2012.

[9] Y. S. Lee and M. W. Cheng, "Intelligent control battery equalization for series connected lithium-ion battery strings." Industrial Electronics, IEEE Transactions on, vol. 52, pp. 1297-1307, 2005.

[10] M. Daowd; N. Omar, P. Van Den Bossche and J. Van Mierlo, "A review of passive and active battery balancing based on MATLAB/Simulink." J. Int. Rev. Electr. Eng, vol. 6, pp. 2974-2989, 2011.

[11] W. Huang and J. A. Abu Qahouq. "Energy sharing control scheme for state-of-charge balancing of distributed battery energy storage system." Industrial Electronics, IEEE Transactions on, vol. 62, pp. 2764-2776, 2015.

[12] R. Ugle, Y. Li and A. Dhingra, "Equalization integrated online monitoring of health map and worthiness of replacement for battery pack of electric vehicles." J. Power Sources, vol. 223, pp. 293-305, 2013.

[13] J. Gallardo-Lozano, E. Romero-Cadaval, M. I. Milanes-Montero and M. A. Guerrero-Martinez, "A novel active battery equalization control with on-line unhealthy cell detection and cell change decision," J. Power Sources, vol. 299, pp. 356-370, 2015.

[14] Y. Wang, C. Zhang, Z. Chen, J. Xie and X. Zhang, "A novel active equalization method for lithium-ion batteries in electric vehicles," Appl. Energy, vol. 145, pp. 36-42, 2015.

[15] United States Advanced Battery Consortium, "Electric vehicle battery test procedures manual." USABC, Jan 1996. 